$\begin{array}{ll} & \text { Etnográfica } \\ \text { etnográfica } & \text { Revista do Centro em Rede de Investigação em }\end{array}$

Antropologia

vol. $19(1) \mid 2015$

Vol. $19(1)$

\title{
La ville dans la ville : sur la densité relationnelle d'un espace public à Lisbonne
}

The city inside the city: on relational density of a public space in Lisbon

\section{Francis Rigal}

\section{(2) OpenEdition}

Journals

Édition électronique

URL : https://journals.openedition.org/etnografica/3920

DOI : $10.4000 /$ etnografica. 3920

ISSN : 2182-2891

\section{Éditeur}

Centro em Rede de Investigação em Antropologia

\section{Édition imprimée}

Date de publication : 1 février 2015

Pagination : 99-120

ISSN : 0873-6561

\section{Référence électronique}

Francis Rigal, «La ville dans la ville : sur la densité relationnelle d'un espace public à Lisbonne »,

Etnográfica [En ligne], vol. 19 (1) | 2015, mis en ligne le 06 mars 2015, consulté le 09 février 2022. URL : http://journals.openedition.org/etnografica/3920 ; DOI : https://doi.org/10.4000/etnografica.3920

\section{(c) (7) \&}

Etnográfica is licensed under a Creative Commons Attribution-NonCommercial 4.0 International License. 


\section{La ville dans la ville: sur la densité relationnelle d'un espace public à Lisbonne}

\section{Francis Rigal}

L'espace urbain à l'intersection de trois places publiques et centrales de la ville de Lisbonne se donne à voir comme un important nœud de rencontres à l'échelle métropolitaine. C'est ainsi un lieu vivant qui accueille toute la dynamique d'une pratique sociale réactualisée dans une tournure transnationale. Sociabilités, rencontres, interactions sont abordées dans cet article afin de décrire une ville relationnelle dans laquelle la diversité des acteurs tend à s'effacer derrière une pratique commune. Et dans un contexte caractérisé par une insistante coprésence, fréquenter la place publique devient une pratique partagée.

MOTS-CLÉS: Rossio, place publique, ville relationnelle, sociabilité, pratique urbaine, transnationalisme.

The city inside the city: on relational density of a public space in Lisbon - The urban space where three public and central Lisbon city squares intersect is seen as an important node of encounters at the metropolitan level. Thus, it is a living space that welcomes the whole dynamic of a social practice in an updated transnational context. Sociability, meetings, and interactions are discussed in this article to describe a relational city in which the diversity of actors tends to disappear behind a common practice. And in a context characterized by a persistent co-presence, attending the public square is shifted into a shared practice.

KEYWORDS: Rossio, public space, relational city, sociability, urban practice, transnationalism.

RIGAL, Francis (antiemail33@gmail.com) - CIES-IUL, Instituto Universitário de Lisboa (ISCTE-IUL), Portugal. 


\section{PRÉSENTATION}

Cet article s'inscrit dans le cadre d'une recherche ethnographique en cours de réalisation qui concerne un espace urbain de Lisbonne à l'intersection de trois places publiques centrales - Rossio, Praça da Figueira et Largo de São Domingos. ${ }^{1}$ Habituellement, les grandes places publiques, en accueillant de multiples pratiques, s'accaparent une pluralité de fonctions qui en font des lieux importants dans la ville. Cet ensemble de places principales de Lisbonne n'échappe pas à la règle et expose aux regards son visage métissé et enchevêtré de vécus quotidiens, comme autant de pratiques qui se juxtaposent, s'entremêlent ou se succèdent. Espace des flux, du repos, de la visite, de la sociabilité, de l'attente, de la consommation ou encore de la rêverie, le lieu est un chaudron d'activités particulièrement variées qui atteste de son caractère multifonctionnel. Face à cette mosaïque de réalités, l'article se propose de témoigner de l'une de ces pratiques sociales, se focalisant sur des citadins qui viennent quotidiennement, ou tout du moins régulièrement, se retrouver au sein de leur réseau d'interconnaissances. Il s'intéresse donc principalement à des habitués qui pratiquent la place dans une dynamique relationnelle, et de fait, la modèlent en un intense lieu de sociabilité. L'analyse a en outre l'intention de rendre compte des qualités de l'expérience urbaine qui se manifestent sur une scène publique dynamisée et marquée par des acteurs dont une grande partie est d'origine étrangère. L'espace présente donc une vitalité humaine caractérisée par une double spécificité, de mixité culturelle et de densité des rapports sociaux. En d'autres termes, il se donne à voir dans une redéfinition contemporaine de la place publique, conjuguant la ville transnationale et la ville relationnelle. ${ }^{2}$

Les trois places publiques de l'espace urbain considéré sont regroupées dans une sorte de configuration en grappe. Rossio et Praça da Figueira sont situés côte à côte, distants d'une cinquantaine de mètres, et connectés par deux liaisons, Rua do Amparo et Rua da Betesga; Largo de São Domingos est adjacent au coin nord-est du Rossio ainsi que relié au nord de la Praça da Figueira par la Rua D. Antão de Almada. ${ }^{3}$ Chacun de ces espaces est donc en lien avec

I Je tiens à remercier la Fundação para a Ciência e a Tecnologia (Portugal) pour sa contribution financière au travers de la bourse SFRH/BD/90782/2012 qui me permet actuellement d'effectuer mon Doctorat en Etudes Urbaines. Je remercie également le Centro de Investigação e Estudos de Sociologia (CIES-IUL) pour son appui en m'accueillant lors de ma candidature pour la bourse d'étude.

2 La formule de ville relationnelle est empruntée à Michel Agier, c'est-à-dire une ville des pratiques citadines et de l'agir commun dans laquelle lien social et expérience de l'altérité sont mis en valeur. Cet auteur se démarque ainsi de l'idéologie de la fragmentation et de la fermeture pour mettre à jour toutes sortes de sociabilités et de créations culturelles nouvelles dans une ville bis et résistante (Agier 1999, 2009).

3 Praça, Largo et Rossio ont des sens à peu près semblables. Ils se différencient moins par leurs usages que par leur apparence et leur fonction. Ainsi, la Praça se particularise par son tracé régulier, [à suivre] 
les deux autres dans une communication circulaire. Du fait de leur proximité géographique, ces trois places sont enveloppées dans le devenir commun de la ville et tendent ainsi à partager un même vécu. Il n'en demeure pas moins que chacune d'entre elles, inscrite dans sa propre histoire socio-urbaine, possède une personnalité singulière. ${ }^{4}$

Le Rossio se présente comme la place principale de la ville. D'une part, il hérite d'une force d'attraction séculaire, d'autre part, au cours de la période moderne, il bénéficie d'une situation privilégiée qui le loge dans le creuset des dynamiques sociales, économiques et culturelles de la vie lisbonnaise. ${ }^{5}$ Du côté sud, il intègre la fameuse Baixa pombaline qui s'étend jusqu'au Tage. ${ }^{6}$ Ce nouveau centre historique, bâti dans un système géométrique méthodique et règlementé pour être une véritable ville moderne, devient à partir du XIX ${ }^{e}$ siècle un cœur économique opérant (Vale 2008). Celui-ci concentre les activités administratives, financières et commerciales de la ville, mais conjointement accueille des artisans, des cafés et plusieurs gargotes. La place du Rossio, située en bordure de la Baixa, côtoie ainsi une population composite à la fois marquée par un tertiaire bourgeois et pénétrée de teintes populaires. Décalé vers l'ouest, sur la colline de São Francisco, bouillonne le quartier du Chiado, devenu entre 1850 et 1950 synonyme de la Lisbonne chic et culturelle avec ses boutiques élégantes, ses cafés, ses clubs et ses théâtres (Salgueiro 2001; França 2002 [1997]). Cette vie de pratiques bohèmes et intellectuelles va alors fortement imprégner le Rossio, notamment en s'installant au sein des grands cafés qui entourent la place et dans lesquels une élite se rassemble pour disserter de littérature et de politique. Puis du nord-ouest, débouchent les habitués mondains de la nouvelle Avenida da Liberdade, artère principale de

sa cohérence architecturale et l'intentionnalité de sa création. Le Largo se distingue alors en tant qu'espace accidentel, un vide ou un élargissement de la structure urbaine. Quant au Rossio, il se caractérise au XIII ${ }^{e}$ siècle comme un terrain public d'usage collectif et proche d'une localité (voir Lousada 2010).

4 La brève description socio-urbaine qui suit concerne une période comprise entre la deuxième moitié du XIXe siècle et les années 80 du XXe siècle. Elle correspond à une époque de significative centralité urbaine de l'espace public dont il est question; une centralité qui s'affirme avec l'extension de la ville vers le nord, et qui se ternit dans un mouvement de décentralisation urbaine.

5 Le Rossio de Lisbonne se précise dès son origine par ses propensions à accueillir la multitude. Au XIVe siècle, l'espace est aux limites de la Lisbonne médiévale, mais il est déjà un point de rencontre fonctionnel par sa situation entre les trois principales composantes urbaines de la ville: Baixa, colline du Castelo et colline de São Francisco. Grâce à ses dimensions, il devient à partir du XV siècle scène des évènements collectifs parmi lesquelles les marchés et les foires, et partage ainsi les activités de la ville avec le futur Terreiro do Paço situé sur les rives du Tage (voir Santana et Sucena 1994; Salgado et Lourenço 2006).

6 En 1755, Lisbonne est presque entièrement démolie à cause d'un tremblement de terre. La situation dramatique est alors prise en main par le Marquis de Pombal qui mènera une reconstruction du cœur de la ville selon un ambitieux projet d'urbanisme moderne, dominé par le rationalisme et la rigueur des proportions. Quelques décennies plus tard, Lisbonne représente la capitale européenne de l'illuminisme (voir França 1989; Vale 2008). 
Lisbonne et scène au début du XX⿳亠丷厂犬⿱ et Martins 1998). La place est cette fois-ci traversée par une promenade à la mode qui parcourt Avenida da Liberdade, Chiado et Baixa. Enfin, dans toute la partie orientale, s'étend l'axe pauvre de Lisbonne, en l'occurrence ses anciens quartiers Mouraria et Alfama, qui enfle le Rossio de sa convivialité populaire: l'immigration rurale vient y vendre des produits de la campagne, les citadins sans travail se retrouvent en de multiples attroupements, et les plus oisifs s'y occupent dans le loisir (Santos 1990; Janeiro 2006). ${ }^{7}$ Il semble par conséquent que ce soit toute la ville, élégante et pauvre, qui est amenée à se rencontrer sur le Rossio. La place apparaît ainsi dans une puissance collective qui en fait un nœud d'articulation entre la Lisbonne libérale et la Lisbonne populaire (Malet 2011). ${ }^{8}$ En d'autres termes, elle endosse un rôle prépondérant de place centrale de la ville. ${ }^{9}$

L'histoire de la Praça da Figueira est beaucoup plus récente. C'est en effet à peine deux ans après le tremblement de terre que naît, sur les décombres de l'Hôpital de Todos os Santos, un marché de fruits et de légumes de la région. Progressivement celui-ci s'agrandit jusqu'à ce que se construise en 1883 un somptueux marché couvert. La Praça da Figueira ${ }^{10}$ incarnera alors, pendant son court siècle d'existence, "o templo sagrado do estômago lisboeta", un lieu où "cada dia começa antes de o anterior ter terminado"11 (Dias 1984: 10-11). En 1949, afin de décongestionner le trafic, mais aussi dans l'intention de moderniser l'image de la ville, le marché est détruit, et avec lui disparaît un quotidien de vie intense et populaire. Tandis que l'Estado $\mathrm{Novo}^{12}$ en fait une

7 Les habitués de la place se souviennent de ces nombreux groupes, disséminés un peu partout, et qui rassemblaient entre 10 et 20 individus. Des rencontres entre gens "que não tinham nada para fazer (qui n'avaient rien à faire)", tel que l'affirme Mário, assis sur son banc. Également, comme se le rappelle Luís (ancien serveur), proche du coin nord-est du Rossio se regroupait tous les matins dans les années 60 un grand nombre d'employés de la restauration pour être embauchés sur le champ.

8 Dans une récente thèse de doctorat, Daniel Malet (2011) est le seul auteur à avoir traité en profondeur du Rossio contemporain. Son analyse, en même temps historique et ethnographique, met en relief la résistance populaire de la place contrastant avec le projet de modernisation de la reconstruction pombaline.

9 Ce rôle de place centrale acquiert peut-être toute sa valeur dans le cadre de la reconstruction pombaline. En effet, dans les intentions de la nouvelle ville, le Rossio était destiné à devenir un lieu secondaire par rapport à sa rivale et complémentaire Praça do Comércio. Et c'est grâce à un tumultueux XIX ${ }^{e}$ siècle d'évènements sociaux et politiques que celui-ci se détourne des prévisions et se réconcilie avec ses fonctions de "verdadeira agora da cidade (véritable agora de la ville)" (voir Santana et Sucena 1994).

10 "Praça" dans le sens de place, et "praça" dans le sens de marché, sont dans la langue portugaise des homonymes. Cela témoigne d'ailleurs de la considérable correspondance entre ces deux réalités. Dans le cas présent, il s'agit pour l'instant du marché de Figueira.

11 "Le temple sacré de l'estomac lisbonnais", où "chaque jour commence avant que le précédent ait terminé”. Les citations seront traduites en note de page, excepté dans les cas d'évidente compréhension. 12 Estado Novo (Etat Nouveau) est le terme officiel désignant la doctrine gouvernementale du régime autoritaire au Portugal de 1933 à 1974. 
place aux héros en érigeant une statue équestre monumentale (1971), l'espace va être successivement animé d'une multitude de prostitués et d'un vivant marché noir dans les années 70, signalant de la sorte le caractère obstiné du lieu.

Le Largo de São Domingos se différencie par sa forme irrégulière propre au tissu médiéval, contrairement aux deux autres espaces précédents, structurés et régularisés par des tracés planifiés. Lieu du peuple semble-t-il, voir des basfonds, décrit au début du XXe siècle par une "atmosfera decadente e boémia, estritamente lisboeta, impregnada dos aromas das iscas e do álcool "13 (Janeiro 2006: 250). Cette allure marginale est sans doute à connecter avec la Rua das Portas de Santo Antão, principale des sept voies d'accès qui mènent à l'espace public, et qui dans les deux premières décennies du siècle dernier est flanquée de clubs de musique et de loisir, attirant ainsi artistes, bohèmes et spectateurs, pour ensuite être considérée quelques décennies plus tard, selon les propos de Mário, comme un "centro de referência da malandragem e da má vida". ${ }^{14}$ Le Largo est de même, à cette époque, directement relié aux quartiers pauvres: placé au pied de la colline de Santana, il reçoit naturellement ses habitants; et situé dans le passage des citadins qui se déplacent vers le centre de la ville, il s'offre comme un lieu transitoire où les plus lisbonnais s'attardent probablement, invités à boire un verre à la Ginjinha,$^{15}$ ou attirés par une certaine animation de quartier dans cet espace plus intime que les deux autres places.

Lépoque moderne atteste ainsi d'un ensemble de vécus qui se répartissent de manière diversifiée dans les trois espaces publics. Et de fait, en étant habités par des pratiques différentes, ces lieux sont assimilés à telle partie du corps social plutôt qu'à telle autre. C'est en effet une population aux mœurs déviantes qui semble particulariser le Largo de São Domingos, avec sa vie de rue et ses excès, alors que la Praça da Figueira, intimement liée à sa fonction nourricière, également lieu du peuple, est d'avantage associée au monde rural. Enfin, le Rossio, en accueillant des milieux sociaux contrastés, se distingue des deux autres espaces par son accointance avec la partie chic de la ville.

Ces divers attributs vont alors fortement orienter les occupations qui aujourd'hui s'exposent sur les places publiques. Ils constituent ainsi le fondement d'une analyse de l'espace et de ses dynamiques d'utilisation. Dans une première partie, il va être question de décrire les occupations spatiales

13 "Atmosphère décadente et bohème, strictement lisbonnaise, imprégnée d'arômes de foie et d'alcool".

14 "Centre de référence de l'escroquerie et de la mauvaise vie".

15 La Ginjinha, ouverte en 1840 (c'est maintenant la cinquième génération du gérant), est une véritable institution de Lisbonne. Le lieu est tout petit, et par conséquent c'est sur le Largo que se consomme l'unique breuvage servi, la ginja, une liqueur de cerises griottes. Paulo, engraxador qui travaille juste devant, raconte les files interminables qui traversaient le Largo de part et d'autre quand la fréquentation était encore plus importante. 
contemporaines, en montrant comment celles-ci, malgré un profond renouvèlement de leur substrat humain, s'inscrivent dans une continuité sociologique. Une deuxième partie aborde l'utilisation de l'espace dans sa dimension relationnelle, envisagée exclusivement à l'intérieur des groupes, c'est-à-dire au sein des réseaux d'interconnaissances. Enfin, une troisième partie s'intéresse aux intersections relationnelles qui s'opèrent entre les groupes. L'espace public se caractérise alors par son expérience urbaine et la perméabilité de ses frontières, témoignant ainsi d'un complexe monde interactionnel qui s'apparente à la ville "réseau des réseaux" mise en valeur par Ulf Hannerz (1983 [1980]).

\section{QUAND LE LOCAL DEVIENT TRANSNATIONAL}

De nos jours, le Rossio, le Largo de São Domingos et la Praça da Figueira présentent une vitalité qui diffère du passé. Cet autre visage procède de mutations socio-urbaines qui ont amené, au cours d'un processus en deux étapes - polycentralisation de la ville et phénomène migratoire - à une redéfinition de la territorialisation des espaces.

Ce changement commence par une intensité urbaine qui ne résiste pas à la décentralisation de la ville s'effectuant progressivement au cours de la deuxième moitié du siècle dernier (Gaspar 1976; Silva 1994; Ferreira 1994; Salgueiro 1997, 2001). Les trois places sont ainsi emportées dans un mouvement de déclin puis de récupération qui affecte toute la zone du centre historique. Le transfert des activités tertiaires vers les quartiers nord, suivi de l'expansion urbaine, avec l'apparition de nouveaux pôles d'attraction de services et de commerces, provoquent dans les années 80 la fin de l'hégémonie de la Baixa en tant que centre des activités économiques. En conséquence, toute cette partie centrale plonge dans une situation d'abandon, aggravée par le vieillissement et la diminution de la population résultant de la dispersion métropolitaine. ${ }^{16}$ Les politiques de la ville réagissent par une volonté de revitalisation commerciale accompagnée d'une réhabilitation de l'espace urbain comprenant les principales places de la Baixa (achevé en 2001). Le Rossio récupère alors l'échelle humaine de son mobilier et se découvre dans une allure hybride, conciliant une réinterprétation de l'existant du XIX ${ }^{\mathrm{e}}$ siècle et une modernisation liée à la mobilité (Fernandes 2001). Praça da Figueira hérite d'un semblable aménagement, partagé entre un désir d'urbanité et une fonctionnalité de la voirie. Le Largo, quant à lui, est entièrement dédié aux piétons. Les trois places se sont ainsi tournées en faveur d'une potentielle vie publique, et la Baixa, qui conserve actuellement une certaine importance économique, est toujours

16 A partir de 1970, la population devient plus nombreuse dans l'aire métropolitaine que dans la ville de Lisbonne, qui perd ainsi son exclusivité démographique. Puis dans la décennie 90, Lisbonne diminue de $25 \%$ sa population (Baptista 1991). 
considérée comme un centre au niveau métropolitain, "numa posição central dos sentidos e das energias"17 (Seixas 2005: 69). ${ }^{18}$ Néanmoins, cette nouvelle situation ne peut masquer l'affaiblissement d'une vie d'autrefois qui résonne encore dans les mémoires. Le lieu n'est plus un "formigueiro"19 tel que le qualifie Paulo, engraxador ${ }^{20}$ au Largo depuis sa jeunesse, ni ce "teatro" qu'évoque Miguel, quand, dans son enfance, il y déambulait pieds nus.

Conjointement à la dispersion de la vie lisbonnaise, de nouveaux acteurs entrent progressivement en scène. Ce processus de substitution, qui en quelque sorte redynamise le vécu des places publiques, est déterminé par deux évènements majeurs. Le premier concerne les retornados, ${ }^{21}$ arrivés dans la capitale en 1975, environ un an après la révolution des œillets. Partis dans l'empressement, ceux qui ne savaient pas où aller se rassemblent avec leur minimum d'affaires personnelles au Rossio et sur la Praça da Figueira. L'Etat va rapidement les loger dans tous les hôtels du centre, mais les deux espaces publics resteront désormais leur lieu de rencontre. En quelques années les occupations s'organisent et l'espace se définit: c'est dans l'allée ouest du Rossio que les retornados se réunissent, ceux de l'Angola, du côté de la Baixa, ceux du Mozambique, du côté de l'Avenida da Liberdade. La place acquiert ainsi une vie intense, fréquentée par plusieurs centaines de personnes, et selon les propos enthousiasmés de Pinto, qui a vécu 32 ans en Angola, "sempre, sempre havia gente, estava sempre cheio" ${ }^{22}$ Les anciens cafés se remplissent également, essentiellement le PicNic, le Passos et le Gelo. ${ }^{23}$ Le lieu fonctionne comme un important espace de ressources dans lequel s'échangent principalement des informations sur la famille et le travail. Il est aussi, dans les premiers temps, une occasion

17 "Dans une position centrale des attentions et des énergies".

18 Un très bref portrait actuel des activités économiques de la Baixa pourrait se caractériser par une centralité préservée dans les services financiers, une certaine continuité dans le commerce en détail de produits de qualité malgré une nette diminution, un renforcement de la restauration, et une spécialisation dans le commerce vestimentaire.

19 "Fourmilière".

20 Engraxador, autrement dit, cireur de chaussures. Ils sont environ une dizaine a travailler, répartis sur les trois places publiques.

21 Retornados, c'est-à-dire la population d'origine portugaise qui face au processus de décolonisation revient au Portugal. Ils sont environ un demi million à se rapatrier en 1975, pour l'essentiel en provenance l'Angola (61\%) et du Mozambique (34\%). Sur le Rossio, la majorité se considère cependant comme réfugié, et non pas comme “retourné”. Parmi eux, il y a aussi les dénommés luso-africanos, des personnes d'origine africaine et naturalisées portugais, pour la plupart des militaires et des fonctionnaires de l'administration coloniale, estimés a 20.000 individus (voir Machado 1994; Pires 2000, 2003).

22 “Il y avait toujours, toujours du monde, c'était toujours plein".

23 Ce serait en grande partie dans la mémoire d'un Rossio central que s'explique l'installation des retornados (voir Gaspar 1987). Cependant, les anciens cafés ont certainement joué leur rôle puisque déjà dans les années précédant 1975, des gens de Mozambique venus pour les vacances ou pour visiter leur famille fréquentaient le Passos, et des étudiants d'origine africaine se retrouvaient au PicNic ou au café Suíça. 
pour vendre de tout, notamment de l'or et des diamants. Les présences vont ensuite, au fur et à mesure, diminuer et quelque peu se redistribuer. Si certains retrouvent la terre de leur famille, ou partent vivre ailleurs, la plupart d'entre eux finissent par décéder. Au début des années 90, les retornados du Mozambique "mudam para" ${ }^{44}$ la Praça da Figueira. L'allée ouest devient alors exclusivement celle des angolais, qui se regroupent principalement devant l'ancien Banco Nacional Ultramarino: "Era de facto o ponto de encontro", se rappelle José qui travaille depuis son enfance dans le kiosque à journaux juste à côté, "e aqui é que se encontravam muito. E quando se vinha à procura de alguém, vinha-se aqui à procura de alguém ". ${ }^{25}$

Le deuxième événement concerne le phénomène de l'immigration en provenance des anciennes colonies portugaises, qui commence à être conséquent à partir des années 80 . L'espace public s'offre alors comme un point de rencontre pour ces nouveaux citadins. Déjà, à la fin des années 60, un premier flux de travailleurs migrants en provenance du Cap-Vert fréquente la Praça da Figueira. Plus exactement, ils occupent son coin nord-ouest, à l'intersection des deux rues qui mènent aux deux autres places, se situant ainsi dans l'articulation des trois espaces publics. Dans la décennie suivante, les présences augmentent et la vitalité du lieu se consolide: "Avant ici, Rossio/Figueira c'était pour les capverdiens comme la Tour Eiffel à Paris", explique Nuchia (propos recueillis en Français), "on ne pouvait pas venir sans y passer ". Sensiblement à la même période, cette intersection accueille également un groupe de "patrícios" ${ }^{26}$ mozambicains et originaires de Goa, qui était encore nombreux il y a quelques années, avant que la plupart ne parte en Angleterre. En ce qui concerne l'établissement des bissau-guinéens sur le Largo de São Domingos, celui-ci débute, semble-t-il, au lendemain des indépendances, quand les anciens commandos des "antigas tropas do ultramar, aqueles que faziam a guerra colonial", ${ }^{27}$ ainsi que le précise Sorry, se rendent au palais Almada pour réclamer leur retraite (Agualusa, Rocha e Semedo 1993; Malet 2011). La présence ne commence cependant à être perceptible qu'à partir des années 80 , puis augmente progressivement et considérablement pour atteindre un pic vers 2005 . Cette territorialisation massive, composée approximativement d'une centaine de personnes, et qui déborde sur le Rossio, s'est distinguée par sa capacité à marquer l'espace, ayant fait de cette zone "a pontinha de África”, 28 ainsi que le fait remarquer Adelino, un capverdien. Enfin, les occupations se sont amplifiées ces dernières années par la présence de migrants non lusophones,

24 "Déménagent".

25 "C'était de fait le point de rencontre, et c'est ici qu'ils se rencontraient beaucoup. Et quand on venait à la recherche de quelqu'un, on venait ici à la recherche de quelqu'un".

26 Ici dans le sens de "gens de la même terre".

27 "Anciennes troupes de l'outre-mer, ceux qui faisaient la guerre coloniale".

28 "La petite pointe de l'Afrique". 
notamment des maliens et des sénégalais, traversant une période d'inactivité professionnelle. D’une manière générale, la fréquentation quotidienne pour la majorité des personnes qui ne sont pas à la retraite a été déterminée par un espace public qui était, dans les années 90, un lieu d'embauche encore fonctionnel. Au cours de la dernière décennie, l'apparition des plus jeunes est ainsi directement liée à une situation d'attente, dans l'expectative d'un travail ou de documents. ${ }^{29}$ Face au manque de perspectives, un grand nombre a réussi à quitter le Portugal pour chercher du travail en Europe, alors que quelques-uns ont préféré revenir dans leur pays d'origine.

Un même processus, le besoin de se rassembler pour de nouveaux acteurs de la ville, a marqué de son emprunte les trois places publiques. C'est d'ailleurs de la sorte que se construit la morphologie d'un espace, façonnée par de successives occupations appartenant à des époques différentes. Et si ces dernières s'accumulent dans le temps, elles évoluent également, se montrant dans des vitalités changeantes. Un état des lieux contemporain se présente par conséquent comme le reflet momentané d'une série d'occupations prises au cours de leur histoire.

Aujourd'hui, le Largo de São Domingos témoigne d'une territorialisation relativement claire qui juxtapose deux périodes. D'un côté un groupe de retraités de milieu modeste et d'origine portugaise qui émane de l'attraction séculaire de la Ginjinha, surnommé par l'un des serveurs "a equipa do copo",30 et autour duquel gravite une foule d'individus. De l'autre côté, les bissau-guinéens organisés autour d'un petit marché qui se déploie en différents points de l'espace et constitué d'une vingtaine de personnes, des femmes qui exposent à même le sol des produits alimentaires africains, et des hommes qui pour l'essentiel vendent de la noix de cola ou travaillent le cuir. Légèrement en retrait, sur les hauteurs du Largo, plusieurs jeunes arrivés récemment, sans travail et quelque peu démoralisés, sont rassemblés dans l'attente d'un meilleur lendemain. L'utilisation de l'espace public s'inscrit donc dans la dualité, partagée entre la continuité d'un loisir populaire et les initiatives commerciales d'une communauté. Toutefois le Largo de São Domingos, toujours aux prises avec la vitalité de son quotidien de rue, manifeste sa singularité à travers ces deux réalités qui apparaissent pareillement comme des pôles importants d'agrégation et de sociabilité circulante.

Les occupations de la Praça da Figueira mélangent également les traces d'un passé et une histoire plus récente. La courte Rua do Amparo accueille

29 Les discours sont explicites à cet égard. Ibrahim, de la Guinée-Bissau: "não há trabalho, por isso as pessoas estão aqui” (il n'y a pas de travail, c'est pourquoi les personnes sont ici); Touré, de la GuinéeBissau: "somos obrigados a vir aqui, não há trabalho" (nous sommes obligés de venir ici, il n’y a pas de travail); Lassana, du Mali: “on est là car il n’y a pas de travail, sinon je ne serais pas ici”... Cette situation commence à partir de l'année 2000 et devient de plus en plus difficile jusqu'à nos jours.

30 "L'équipe du verre". 
pendant toute la journée les allées et venues d'un ensemble d'individus de milieu populaire qui laisse deviner le souffle encore flottant de l'ancien marché par la présence d'un étalage ambulant, de la criée des vendeurs de loterie, et de quelques personnes adossées au mur et égarées dans leur solitude. Au cours de l'après-midi, à l'intersection de cette ruelle avec la place, se croisent une poignée de retornados du Mozambique, un petit groupe de retraités originaires de Goa, quelques individus de la Guinée-Bissau ou du Sénégal, ainsi qu'une vingtaine de capverdiens, "amigos e conhecidos" 31 aux milieux sociaux diversifiés. Décalée sur les bancs, dans le calme ombragé de la façade ouest de la place, une population en correspondance avec la Rua do Amparo se mêle avec des retraités africains de différentes nationalités. La Praça da Figueira est, par conséquent, un lieu fréquenté surtout durant la deuxième partie de la journée en deux endroits distincts: une partie regroupant l'intersection et la petite rue de jonction, qui privilégie le dynamisme des rencontres, et une partie, à l'écart, qui fonctionne pour certains comme une arrière salle de l'ensemble de l'espace public. "Figueira" se démarque sans doute des deux autres places par la mixité des occupations, donnant à voir une mosaïque de groupes hétérogènes qui se côtoient dans une relative proximité. Ces utilisations emmêlées révèlent la disponibilité d'un espace en train de renaître de ses cendres.

Au Rossio, les utilisations se distribuent sur les deux allées principales qui sont séparées par la circulation et un grand parvis central. Afonso, retornado d'origine angolaise, connaisseur de la place depuis son adolescence, définit l'allée ouest comme le lieu des angolais. En effet, d'une part il accueille "o fim duma cultura", 32 tel que le souligne un ancien pilote d'avion, c'est-à-dire une petite trentaine de retornados, âgés de 70 à 90 ans, qui sont installés quotidiennement sur les bancs pendant quelques heures, en début ou en fin d'après-midi, et répartis en "círculos de amizades" ou en "grupos de amigos". ${ }^{33}$ D'autre part, l'espace est continuellement parcouru par des angolais de classe sociale tendanciellement élevée. Tel que le révèle avec insistance Mogas, mécanicien dans l'aviation durant 35 ans de guerre: "Aqui é um lugar de passagem onde passam todos os angolanos". ${ }^{34}$ Cette allée est cependant aussi fréquentée par quelques bissau-guinéens désirant s'écarter d'un Largo qualifié comme "um pouco de confusão", ${ }^{35}$ ainsi que par un groupe d'une douzaine de retraités homosexuels qui se retrouvent tous les jours en fin d'après-midi. Ce groupe,

31 "Amis et connaissances".

32 "La fin d'une culture".

33 "Cercles d'amitiés", "groupes d'amis".

34 "Ici c'est un lieu de passage où passent tous les angolais". En effet, cette allée ouest ressemble à un corridor de tous les angolais de Lisbonne: des généraux à la retraite, un directeur de la poste à Luanda, d'anciens partisans de l'indépendance... Et quand arrive en voiture un couple venu de l'Angola pour un week-end, c'est au pied de cette allée qu'il est déposé.

35 "Un peu de confusion". 
constitué il y a environ dix ans, s'est établi à l'extrémité nord de l'allée qui était déjà, tel que le raconte Lucas du haut de ses 82 ans, un lieu de rencontres masculines au début du XXe siècle. De l'autre côté, l'allée orientale accueille pendant la matinée un groupe d'une petite dizaine de maliens, les plus assidus de la communauté capverdienne, et des bissau-guinéens qui occupent les bancs proches au Largo de São Domingos ainsi que le muret du théâtre. Au cours de la journée, ces derniers animent le lieu par l'intense sociabilité d'une présence atteignant régulièrement la cinquantaine de personnes, en général des anciens combattants à la retraite mêlés à une migration de réfugiés appartenant à une relative élite sociale. Pendant l'après-midi, s'installent également dans l'allée un groupe d'une quinzaine de jeunes sénégalais d'un milieu social plutôt aisé qui se retrouvent autour d'un banc ou contre le muret de la sortie du métro, puis un petit noyau de retornados de l'Angola, extension de l'allée opposée, provoquée il y a deux ans à peine suite à un conflit interpersonnel. La multitude des occupations qui parcourt le Rossio montre ainsi une place qui non seulement se réaffirme dans une vigueur élargie de sa centralité, mais aussi qui continue de se distinguer des deux autres espaces publics par sa relative appropriation de milieux sociaux privilégiés. Une convivialité de rue dispersée dans une pluralité d'origines, une sociabilité de boulevard incarnée par la bourgeoisie angolaise, font cependant du Rossio un espace intimement pris dans une réalité migratoire.

La chronique de ces trois espaces a mis en évidence l'apparition sur la scène publique de nouveaux citadins alors que la vie urbaine, auparavant concentrée, s'est dispersée dans la métropole. Autrement dit, même si l'intensité n'est plus celle du passé, ces trois espaces se sont réactualisés par la tournure cosmopolite de leurs acteurs, et sont devenus des lieux de confluence éminemment transnationaux, pris dans les flux de la globalité. C'est d'ailleurs une constante dans le devenir des espaces urbains centraux que d'évoluer en étroite connivence avec les mutations de la ville. Cependant, les effets de cette récente période de chamboulements doivent être mitigés. Car non seulement le dynamisme du lieu se perpétue, mais aussi les personnalités historiques propres à chaque espace se devinent encore aujourd'hui, comme si la nouvelle donne humaine s'était tendanciellement insérée dans des utilisations socialement déjà tracées. Le quotidien contemporain des places publiques se laisse par conséquent comprendre dans une continuité renouvelée de leurs principales caractéristiques.

\section{LES RÉSEAUX DE PLACE: RENCONTRES ET SOCIABILITÉS}

L'espace public, tel qu'il se présente aujourd'hui, se distingue par la densité sociale qui l'habite, conjuguant hétérogénéité de ses acteurs et intensité de ses rencontres. Une première description de ce monde relationnel a montré un ensemble d'occupations différenciées dans le temps, et par conséquent elle a 
permis de rendre compte d'un espace public composé dans une diversité des logiques d'utilisation. Cependant, en portant l'attention sur la pratique relationnelle, l'espace se révèle également homogène, uniformisé par un ensemble de caractéristiques communes à tous les groupes. Autrement dit, la similarité des pratiques transcende l'hétérogénéité des occupations. C'est en effet dans une même logique que rencontre des connaissances et sociabilité de groupe se déploient dans le quotidien des trois places publiques. ${ }^{36}$

En abordant la dynamique relationnelle au sein même des réseaux d'interconnaissances, se manifestent plusieurs modalités de la rencontre auxquelles s'expose l'ensemble des acteurs qui régulièrement fréquentent la place. Il est ainsi possible de décliner quatre types de rencontre, déterminés par différents niveaux de fréquentation, transversaux aux groupes, et témoignant d'une variété de circonstances. Premièrement, la rencontre présupposée, qui concerne principalement le noyau dur des réseaux, "a velha guarda", ${ }^{37}$ en fait des habitués de longue date, mais qui intègre aussi des connaissances peutêtre plus récentes et comprises dans le quotidien. C'est la rencontre qui a de fortes probabilités de se réaliser car les acteurs sont pratiquement tous les jours sur la place. Deuxièmement, la rencontre prévisible, qui se produit quand apparaissent des acteurs qui fréquentent le lieu mais de façon irrégulière. Ces habitués inconstants, en bordure temporaire, viennent se recoller à leur réseau de place en des périodicités très variables. Troisièmement, la rencontre occasionnelle, se déroulant au contact d'acteurs périphériques, en quelque sorte des électrons libres qui ont connaissance du lieu, soit pour l'avoir pratiqué par le passé, soit pour en être informé. Ce type de rencontre, relativement répandu et dont les protagonistes sont en général de passage à Lisbonne ou viennent juste d'y arriver, met en relief la puissante source de renseignements que constitue la densité relationnelle des réseaux. Il atteste également du rôle urbain de la place publique en tant que nœud de rencontre transnational. Quatrièmement, la rencontre contingente, soumise au hasard, se démarque de la précédente en étant dénuée d'intentionnalité, et met en relation un habitué avec une connaissance qui appartient à un réseau extérieur de la place. Peuvent alors survenir, en ces momentanés croisements de réseaux, de brusques modifications de rôle. La place publique apparaît ainsi comme un lieu propice où se manifeste la fluidité de la vie urbaine (Hannerz 1983 [1980]), c'est-à-dire

36 La méthodologie de la recherche a été dominée par l'intention d'intégrer chacun des groupes. Se sont ainsi instaurées des relations de confiance dans lesquelles il a été possible de prendre des notes sur le vif. L'observation de l'intérieur s'est donc enrichie d'informations récoltées non seulement au cours du quotidien, mais aussi par de multiples entretiens informels. Une connaissance générale de la place publique, une dizaine d'entretiens approfondis et enregistrés, et un recueil de caractérisation des acteurs (âge, métier, domiciliation, fréquentation de l'espace), complètent les données du terrain. 37 "La vieille garde". 
cette disponibilité personnelle de changer en fonction des relations, quand par exemple passe à l'improviste un collègue de travail, ou un voisin de pallier.

Cette rapide description montre comment, se rendre sur la place publique, c'est aussi aller à la rencontre de connaissances qui ne sont pas forcément prédéterminées. En ce sens, elle permet d'appréhender une double fonctionnalité au sein des réseaux: la dynamique interne du réseau restreint, inscrite dans le quotidien et dans laquelle se renouvèlent les liens et se réaffirment les appartenances; l'ouverture d'un réseau élargi, qui rayonne sur plusieurs échelles selon la nature des groupes, en même temps disponibilité extérieure et point de référence. ${ }^{38}$ L'articulation de ce modèle ne doit cependant pas être interprétée dans un rigide cloisonnement. Une rencontre apparemment contingente peut éventuellement s'avérer intentionnelle, et une rencontre présupposée manque parfois son rendez-vous. De même, chaque acteur est susceptible de changer son mode de fréquentation. Par conséquent, les réseaux qui tendanciellement sont stables, se trouvent en quelque sorte dynamisés par ces rencontres inscrites dans l'éventualité. Les groupes se modèlent ainsi, au quotidien comme sur la durée, dans un renouvèlement variable de leurs acteurs.

La place publique, espace des rencontres, est corrélativement lieu d'une intense sociabilité. "Tagarelar", "encher o papo de conversa", "dar à língua", 39 autant de termes pour signaler l'enthousiasme du bavardage et le bien-être dans l'oralité des échanges. Cette convivialité de place, c'est d'abord dans une dimension ludique qu'elle se montre, de façon répandue et encouragée par les affinités de groupe. Une sociabilité telle que la caractérise Georg Simmel (1981 [1970]), pour elle même, quand le plaisir est dans l'intention unique d'échanger des propos. Les causeries se nourrissent alors d'anecdotes, de ragots et de commentaires futiles, et les interactions s'épanouissent au sein des réseaux indépendamment de la qualité des liens et de l'intensité des fréquentations. Mais les conversations possèdent aussi une dimension sociale, traitant de sujets plus sérieux ou plus intimes. La sociabilité, tournée vers l'échange d'opinions ou vers la transmission d'informations, participe ainsi par ses discussions et ses propos personnalisés à consolider les liens entre les acteurs. ${ }^{40}$ Bien qu'un fond d'homogénéité culturelle accompagne la plupart des bavardages (football et

38 Il y a des acteurs de la sorte, qui passent des heures sur leur banc, avant tout pour le plaisir des rencontres inhabituelles. De même, il n'est pas rare d'observer des personnes qui au cours d'une interaction sont aux aguets d'une rencontre occasionnelle.

39 "Causer", dans le sens de parler trop et avec indiscrétion, "remplir l'estomac de conversation", "donner la langue".

40 En fait, la qualité des liens d'interconnaissance varie considérablement à l'intérieur de chaque réseau. S'ils s'activent et s'entretiennent dans la répétition des rencontres, ils sont aussi déterminés par d'autres contextes. Connaissance de place, camarade du service militaire, natif d'une même terre, ami d'enfance, membre de la famille, c'est toute une variété de réalités qui structure les rapports au sein des groupes. 
actualité politique), la pratique discursive est contrastée par des centres d'intérêts propres à chaque groupe. Mais en même temps, elle se caractérise par sa nature fondamentalement transnationale. Car les thématiques exprimées sont avant tout déterminées par des contextes géographiques de toutes sortes. Les mésaventures d'un frère éleveur aux environs de Bamako, les qualités d'un candidat aux élections en Guinée-Bissau, l'expansion urbaine de Lobito, ou encore le climat de Londres, s'offrent ainsi, au grès d'une promenade dans l'espace public, comme des bribes de discours qui parcourent le monde. Enfin, la sociabilité de place comporte une forte dimension utilitaire. Dans chaque groupe, les acteurs bénéficient mutuellement des compétences de chacun par l'échange de renseignements précis ou de petits services en tous genres, et il est aussi relativement fréquent que s'exposent des soucis financiers afin de profiter d'un prêt ou d'un don. Cette sociabilité de l'entraide se renforce auprès de certaines figures qui s'occupent de "tratar"41 de démarches administratives. Courants dans les divers réseaux en lien avec l'immigration, ces acteurs facilitent l'obtention de documents dans un échange généralement symbolique. La sociabilité de l'espace, succinctement abordée, renvoie ainsi aux différents aspects de la fonction relationnelle d'une place publique, simultanément lieu de la plaisanterie, lieu du débat, et lieu de ressources socio-économiques.

Dans la régularité des rencontres qui chaque jour se réactualisent et d'une sociabilité sans cesse rejouée, tel un véritable rituel urbain de l'espace public, s'élabore une intime familiarité avec le lieu. Venir au Rossio confère alors un agir commun à l'ensemble de ces habitués. Ces acteurs de place, José les distingue des gens de passage en parlant de "a gente do Rossio", puis il les caractérise tout simplement: "Tu consegues reconhecê-los só pela forma como se mexem ". ${ }^{42}$ En effet, les gens du Rossio entretiennent avec l'espace un rapport de familiarité qui s'exprime par une aisance généralisée dans les manières d'être. La désinvolte sérénité d'une position assise, la nonchalance d'une déambulation, ou encore une attente qui se perd dans la flânerie, témoignent de la liberté d'esprit qui s'approprie des postures et des mouvements. Ce bienêtre dans l'espace s'observe également par la fluidité des occupations. Quand l'ici est mouvant: un groupe qui s'éparpille au grès des discussions, ou qui se décale par de successifs déplacements en échos des humeurs et des commentaires. Simultanément, une certaine volatilité des présences signale une maîtrise sans contrainte de son environnement. La pratique relationnelle est ainsi souvent accompagnée d'activités personnelles qui génèrent de soudaines transformations des groupes qui se font et se défont en fonction d'un tel qui va à un rendez-vous ou de tels autres qui vont faire leurs courses ou boire un café. La familiarité avec l'espace, doublée par la familiarité avec ses connaissances, 
se manifeste également par une certaine sociabilité libérée au cours des interactions. Il est ainsi commun que les récits d'évènements ou les histoires anecdotiques se transforment en terrain fertile sur lequel la sociabilité prend des allures théâtrales. Le groupe devient alors public et l'acteur, emporté par le contexte, transforme la narration en une cocasse représentation.

Mais ce sont certainement tous les gens du quotidien qui se proposent en spectacle. Car ces façons d'être à l'aise, ce naturel en compagnie comme dans la solitude, fait de la place publique un espace d'intimité pour ses habitués. Quand, par exemple, le Senhor Silva lit placidement son journal, impassible au milieu des flux et des coprésences, cette présence s'apparente à un chez-soi posé au cœur de la ville. Par les innombrables vécus de citadins qui viennent chacun à leur manière faire leurs petites emplettes, le lieu se montre comme l'espace du privé collectif. De la sorte, privé et public semblent se brouiller, c'est-à-dire que leurs territoires respectifs tendent à se confondre. D'ailleurs, les acteurs jouent de cet espace ambivalent, usant des privilèges de la sphère intime comme des avantages d'une ouverture sur le monde. Et de fait, toutes ces intimités s'exposent à l'arène publique. Elles seraient “en vitrine", tel que le ressent Acácio, lorsqu'il est assis sur un banc au milieu de ses camarades "un peu tous fous". La pratique de place pourrait alors être considérée dans sa routine quotidienne telle une mise en public du privé.

\section{QUAND LA VILLE RELATIONNELLE SE CONCRÉTISE}

L'espace public s'affiche ainsi dans son rôle urbain éminemment public de lieu de rencontre, accueillant des citadins qui partagent une partie de leur quotidien au sein de leur réseau d'interconnaissances. Toutefois, la sociabilité de place n'est pas confinée à l'intérieur des groupes. Au contraire, la vitalité des rencontres déborde les réseaux de chacun, et une lecture croisée de la place relationnelle dévoile la perméabilité des frontières entre chaque petit monde. L'espace public est alors celui de l'expérience urbaine, espace de potentialité de la rencontre dans un contexte de proximité, autrement dit un espace dans lequel il est possible de se frotter à quelqu'un qui n'est pas soi-même (Joseph 1995). Ainsi, la sociabilité, en se dilatant dans un éparpillement des interactions, modèle une place qui tend à concrétiser la ville relationnelle. Il serait toutefois opportun de considérer la place publique dans son contexte élargi, comprenant également tous ceux qui ne font que passer, et qui constituent, sur la durée, la partie majoritaire des présences. ${ }^{43}$ Ce décor mouvant et quotidien,

43 La métropole qui travaille, traversant un espace central nœud de la mobilité. Une autre métropole qui foule les trottoirs au cours d'une promenade, ou qui se repose sur un banc dans un parcours d'activités. Mais aussi, et plus récemment, des essaims de touristes qui ponctuent leurs déplacements par de stratégiques arrêts, ou encore des groupes disparates et de toutes provenances (équipes sportives, employés d'entreprises, bandes d'amis) qui animent l'espace par leur nature singulière. [à suivre] 
dans le hors échelle d'une pratique de la ville, offre en effet de multiples occasions de rencontres surprenantes et spontanées.

L'expérience urbaine s'inscrit en étroite correspondance avec les qualités de l'espace lui-même. Car un ensemble de caractéristiques singulières à ces places en fait un espace prédisposé à la rencontre. Ainsi, la présence de la Ginjinha sur le Largo de São Domingos, la patine symbolique de l'ancien marché de Figueira, et la capacité du Rossio, déjà évoquée précédemment, à incarner la diversité lisbonnaise, participent à doter l'ensemble de l'espace public d'une vocation collective. Ce caractère s'affirme d'autant plus que l'espace, dépourvu d'architectures significatives du pouvoir, respire dans une certaine indépendance fonctionnelle. Car les places sont principalement bordées par des bâtiments résidentiels, et les quelques édifices institutionnels présents, Teatro D. Maria II, église de São Domingos et Palácio Almada, sont dénués d'efficacité symbolique d'appropriation, contrairement à leur voisine Praça do Comércio, fortement marquée par les fonctions administratives de son édifié et par sa configuration de place royale. Une allégorie du pouvoir s'installe pourtant avec ostentation en 1870 lorsque s'élève au centre du Rossio, rebaptisé pour l'occasion Praça D. Pedro IV, une statue royale sur une colonne monumentale. Mais l'autonomie de la place résiste. Aujourd'hui, c'est toujours par le terme de Rossio que les habitants continuent de nommer le lieu. Quant au monument, il voit sa puissance évocatrice détournée par une croyance en la substitution de la statue officielle par celle de l'empereur du Mexique, Maximilien I. À cette inclinaison collective, s'ajoute le penchant fédérateur d'un lieu interface. En effet, l'espace de l'entre deux, ou encore de l'intervalle, génère dans ses interstices une sorte de vide sociologique propice à stimuler les relations (Agier 2009). Et l'ensemble des places, à cet égard, est situé géographiquement en de multiples intersections de la ville: à la confluence de plusieurs quartiers, du fait de sa situation topographique l'ayant installé au pied de trois collines et en bordure de la Baixa; au seuil d'une rupture entre la ville médiévale tournée vers le Tage, et la ville moderne qui se déploie vers l'intérieur des terres; au centre d'un axe, tel qu'il a été auparavant indiqué, qui articule la ville libérale du côté occidental et la ville populaire du côté oriental. Par conséquent, l'espace se propose pourvu de potentialités collectives et unificatrices, se laissant de la sorte définir par ses vertus publiques, à la fois à tout le monde et à personne.

Le monde relationnel de l'expérience urbaine ne peut également être abordé sans prendre en considération un double déterminant inscrit dans une quotidienneté de l'espace public. Premièrement, ceux qui viennent sur la place

[suite] Cette pratique de groupe est sur la place abondante et omniprésente, variant selon les périodes de l'année. Son importance résulte d'un contexte favorable. D’une part, Lisbonne fait partie d'un cluster transnational de capitales touristiques à voir. D'autre part, le Rossio est le passage obligé parmi les parcours principaux de la ville. 
se rencontrer sont confrontés à une situation de promiscuité avec d'autres acteurs. En effet, la localisation des réseaux étant généralement stable, l'espace présente un ensemble de groupes aux proximités variables, mais qui se juxtaposent selon un schéma répétitif. Cependant, les déplacements et les éparpillements de certains d'entre eux entraînent un aspect quelque peu emmêlé de ces occupations. Les acteurs sont par conséquent exposés à un contexte de coprésence défini par des habitudes et qui en même temps laisse la place à des altérations de voisinage. La place est ainsi investie d'une myriade de promiscuités possibles, se réorganisant au cours de la journée et amplifiées par la présence de quelques autres groupes qui fréquentent le lieu aussi quotidiennement mais pour des logiques différentes (gitans, animateurs de rue, clochards). La Praça da Figueira est de ce point de vue explicite, avec ses bancs régulièrement partagés, et son intersection qui en des moments de la journée peut être un lieu de rencontre de trois ou quatre réseaux simultanément. Pareillement, il arrive occasionnellement que sur un même banc du Rossio s'installent jusqu'à trois petits groupes différents, transformant le mobilier urbain en un îlot d'occupations disparates et serrées. Cet effet de proximité, qui en certains lieux est plus évident, s'offre alors comme des occasions de rencontres fortuites dans lesquelles se créent des étincelles de connexion. Deuxièmement, le facteur du temps, dans une routine qui se prolonge parfois sur de nombreuses années, assoupli les frontières et cultive le terrain des rencontres. En d'autres termes, une sorte de fatalité de la durée permet aux relations de se nouer plus facilement. "Com o tempo... devagarinho", ${ }^{44}$ indique Aquino, un retraité de Goa, pour expliquer son interaction avec Adriano qui fait partie de la clique de Rua do Amparo. "Venho aqui há muitos anos", signale cette autre personne de Mozambique pour justifier de ses multiples liens avec tant de monde. "Vejo-os todos os dias, conheço toda a família”, raconte José Luís, cet engraxador qui discute souvent avec les gitans. Les liens qui se tissent dans l'espace public se rapportent ainsi directement à un processus d'insistante familiarité dans lequel se complémentent la proximité des acteurs et l'accumulation des fréquentations. Cette familiarité agit par conséquent comme un véritable générateur de liens entre des acteurs appartenant à des réseaux différents.

Au gré des rencontres, certains acteurs entretiennent des liens privilégiés, tandis que d'autres, à travers une ancienne conversation ou un échange anodin, se connaissent à peine. ${ }^{45}$ Circonscrite aux interactions, l'interconnaissance concerne une partie des habitués, mais prise dans un sens large, elle irrigue l'ensemble de la place. Ainsi, pour avoir été maintes fois aperçus, pour avoir été

44 "Avec le temps... peu à peu", puis dans les phrases suivantes, "Je viens ici depuis de nombreuses années", "Je les vois tous les jours, je connais toute la famille".

45 Ces relations de place se caractérisent généralement par un lien de qualité publique, et qui pourrait se résumer par le commentaire désinvolte de Monsieur Figueira: "eu conheço-o daqui; não sei como se chama (moi je le connais d'ici; je ne sais pas comment il s'appelle)”. 
aussi minutieusement observés, ou pour avoir été renseignés, les savoirs et les représentations entre les habitués se répandent inexorablement et repoussent les frontières de l'anonymat. La connaissance des autres est d'ailleurs régulièrement mise en valeur, souvent évoquée, tel l'indice d'une compétence sur le lieu, et parfois emballée dans une impression du "conheço todos" " ${ }^{46}$ Ces diverses attitudes seraient alors à interpréter comme la promotion d'un espace idéalisé de l'interconnaissance (Viera 1998). De fait, l'anonymat tend à ne pas vouloir trouver sa place. Car dans une situation d'incessante coprésence, peutêtre que les acteurs ne se connaissent pas vraiment, mais tout du moins ils se reconnaissent. Se reconnaître, c'est-à-dire se considérer, dans un mécanisme de mutuelle familiarité, comme appartenant à la "gente do Rossio", équivaut à dévoiler ce lien minime d'interconnaissance autour d'une unique certitude: l'Autre est pareillement quelqu'un qui vient pratiquer l'espace public.

La place, en tant qu'espace public qui est à tous, témoigne par conséquent de ses qualités urbaines pour devenir l'espace de l'entre tous. Espace de connexions entre les groupes, espace de chevauchements entre les réseaux. Corrélativement, ces intersections s'articulent en des variabilités aussi bien quantitatives que qualitatives, et engendrent un cadre interactif quelque peu confus. Cependant, il serait possible de caractériser la dynamique des interactions en distinguant plusieurs niveaux d'intersections en fonction des densités relationnelles mises en jeu. Les intersections peuvent ainsi être singulières, ce sont les plus fréquentes, et elles s'activent quand interagissent deux acteurs de réseau différent. Elles peuvent toutefois présenter des qualités plus profondes, quand un acteur entre en relation avec plusieurs acteurs d'un autre réseau. ${ }^{47}$ Il est aussi possible, mais les cas sont plus rares, qu'un habitué de longue date étende les intersections vers plusieurs acteurs et dans plusieurs réseaux à la fois. Ces figures de la place s'apparentent alors à des plateformes de liens sociaux et se comportent comme des charnières relationnelles. Enfin, les réseaux sont parfois un peu enchevêtrés entre eux. La frontière entre deux groupes s'est délitée et plusieurs acteurs d'un groupe entretiennent des liens d'interconnaissances par des interactions individuelles avec plusieurs acteurs d'un autre groupe. Les capverdiens et la clique de Rua do Amparo, par exemple, qui se côtoient quotidiennement, ou encore un petit groupe de retornados de l'Angola et un autre de la Guinée-Bissau, qui papotent dans la même zone, témoignent de ce type

46 "Je connais tout le monde".

47 Plusieurs exemples peuvent être cités: José, qui tous les matins quitte son groupe de clochards pour s'installer avec la clique de la Ginjinha; César, cet ancien du Mozambique, qui parfois s'assoie en compagnie de bissau-guinéens; Dabo, de la Guinée-Bissau, qui chaque matin s'arrête quelques instants au banc des maliens; Alexandro, un commerçant, qui discute régulièrement devant sa boutique avec plusieurs musiciens de rue; Paulo, engraxador sur le Largo de São Domingos, salut de nombreux bissauguinéens; Sorry, de la Guinée-Bissau, qui s’arrête de temps à autre avec des retornados de l'Angola... 
d'intersection démultipliée. Les acteurs de l'espace public établissent ainsi, en des proportions variées, des liens de toutes sortes en dehors de leur réseau.

Sans aucun doute, ces connexions sont favorisées par la dimension transnationale des acteurs eux-mêmes. En se penchant sur les parcours de vie, se découvrent alors, dans une proportion qui semble relativement élevée, des existences en marge du modèle commun. Les récits sont en effet tendanciellement extravagants et rocambolesques. Mécanicien de la marine marchande, mercenaire, garde du corps de présidents, soudeur d'oléoduc, fabricant de faux billets de banque, prospecteur de pétrole, fusilier dans les troupes coloniales ou encore bohémien saisonnier, c'est un visage de la place qui se dévoile dans une population de vieux aventuriers dont les expériences de vie s'étalent en des énumérations de pays interminables. Des existences par conséquent marquées par de perpétuels déracinements, et en même temps porteuses d'un complexe puzzle identitaire. Et c'est certainement la majorité des acteurs de la place, pour la plupart retornados, luso-africains et migrants, qui est imprégnée de ces identités-fusions. ${ }^{48}$ Des anciens de Goa, dont l'identité se répartit sur trois continents, suite à de successives situations de réfugiés; des bissau-guinéens, dont certains ont étudié à Dakar, puis suivi des formations en Russie ou en Algérie, pour enfin fuir leur pays et venir à Lisbonne; des retornados, dont la double identité se complexifie souvent de vécus en Afrique du Sud, en Namibie ou encore au Brésil. Ces exemples, parmi tant d'autres, soulignent une relative homogénéité des constructions identitaires. Car elles sont pourvues de qualités cosmopolites, inscrites de plein pied dans les dynamiques de la globalisation, et façonnées par une routine transnationale. Les connexions entre les acteurs s'établissent par conséquent sur un terrain nivelé. Elles sont en quelque sorte rendues disponibles. Et la place publique devient ce lieu aux frontières assouplies, espace de l'occasion des échanges, dans lequel se racontent des souvenirs militaires, se confrontent des expériences de travail au Moyen-Orient, se relatent des péripéties dans la mer du Nord.

La pratique de place prend ainsi du sens. L'espace relationnel apparaît en effet comme un moyen pour les acteurs de la mobilité d'aller à la rencontre de leur propre histoire. C'est d'ailleurs l'espace public pris dans sa totalité qui fonctionne comme une caisse de résonance des existences de chacun - ce "campo do maravilhoso que só existe aqui" ${ }^{49}$ tel que le remarque pertinemment José, une scène où " todo o mundo passa" ${ }^{50}$ dont la diversité ne peut être décrite mais seulement évoquée par des passages au hasard, celui d'un guide égyptien, de rugbymen ukrainiens, d'une famille saoudienne, d'un groupe de

48 Pour rendre compte du précipité urbain dans le multiculturalisme d'une Lisbonne contemporaine, Cristiana Bastos (2001) propose le terme de fusão, préféré à celui de globalisation. Il s'agit de mettre en évidence un brouillage des identités, dans une réalité où tout semble s'imbriquer.

49 "Champ du merveilleux qui n'existe qu'ici".

50 "Tout le monde passe". 
pharmaciens français, ou encore, d'un personnage extravagant. Prendre place dans un contexte et se laisser prendre par de potentielles sociabilités, renvoie aux acteurs, par de multiples références, la saveur du vaste monde. La densité relationnelle de la place publique s'articule alors autour d'un lien complice entre les acteurs et l'espace: car se répondent, dans leurs mutuelles ouvertures, des citadins aux larges appartenances et la dimension cosmopolite d'une ville.

Et c'est bien une majorité d'entre eux qui se montre disposée à faire de nouvelles rencontres. En ce sens la place publique se présente comme la ville des communautés qui se côtoient et se fréquentent. Elle serait alors consacrée dans sa dimension symbolique, affichant sa vocation de mettre ensemble le corps social dans une quête du vivre ensemble (Ghorra-Gobin 2001). Cependant, celle-ci n'est pas pour autant un espace de l'idéal généralisé de l'interculturel. L'expérience urbaine de la place est en effet légèrement enrobée par de petites doses d'hostilité, concernant les considérations sur les autres, et qui entachent la création de liens. Néanmoins, de multiples situations témoignent d'interactions spontanées qui réussissent à surprendre les inimitiés, et montrent en même temps comment ces dernières finalement s'accommodent des relations d'interconnaissances instaurées. Juger de l'expérience urbaine de l'espace public relève par conséquent d'une mesure qui se perd dans l'hétérogénéité des acteurs. Car ceux-ci, également, se contentent de leur propre monde relationnel. Et si d'une part l'espace est effectivement le lieu où les réseaux tendent à se croiser, d'autre part la densité relationnelle, mitigée selon les intentions de chacun, louvoie entre l'entre tous et l'entre soi.

\section{POUR CONCLURE}

Où en est l'espace public d'aujourd'hui ? Ce lieu dont le visage habituel s'est modifié par un renouvèlement de ses acteurs. Ce lieu encore, qui résiste à la ville desserrée, continuant à occuper une place privilégiée dans les représentations en tant que "coração da cidade",51 alors que le tissu urbain de la métropole est désormais marqué par un réseau de centralités. Se détache la constatation d'un espace public bien vivant, irrigué par une convivialité quotidienne où prédomine le rôle d'une population de migrants. Et c'est certainement en considérant cet espace qui se pratique dans le loisir, notamment pour ses retraités, s'offrant selon le bon vouloir et le plaisir de chacun, que se révèle le lieu, car celui-ci se démontre, et peut-être encore plus qu'avant, comme étant voué au service des individus. Il s'agit d'un lieu qui est manifestement utilisé dans une logique communautaire, à la fois espace de ressources et nœud de rencontre en même temps local et transnational. Corrélativement un lieu du collectif, et 
par conséquent scène tolérante, qui fonctionne d'une certaine façon comme un refuge pour des acteurs sujets à la discrimination. Migrants, retornados, homosexuels, pour cette population en marge de la société, la scène urbaine principale apparaît comme l'endroit du possible afin d'incorporer la ville. Ce lieu est également théâtre cosmopolite, c'est-à-dire d'une identité difficilement saisissable, à la fois neutre et éparpillée, qui se donne comme un espace d'identification pour ces acteurs citoyens du monde. Finalement la place publique, dans sa destinée à s'occuper du corps social, se précise aujourd'hui par une fonction intimement citadine: être ce lieu où il est possible de se relier aux autres. N'est-ce pas là, en effet, que les individus font la ville?

\section{BIBLIOGRAPHIE}

AGIER, Michel, 1999, L'invention de la ville: Banlieues, townships, invasions et favelas. Paris, Editions des archives contemporaines.

AGIER, Michel, 2009, Esquisses d'une anthropologie de la ville: Lieux, situations, mouvements. Louvain-la-neuve, Academia Bruylant.

AGUALUSA, José E., Elza ROCHA, et Fernando SEMEDO, 1993, Lisboa Africana. Porto, Edições Asa.

BAPTISTA, Luís V., 1991, O Estado Novo e o Programa das Casas Económicas em Lisboa. Oeiras, Celta Editora.

BASTOS, Cristiana, 2001, "Omulu em Lisboa: etnografias para uma teoria da globalização", Etnográfica, V (2): 303-324.

DIAS, Marina T., 1984, "Praça da Figueira: memória duma cidade alegre", Diário Popular, 7 décembre, 10-11.

FERNANDES, José M., 2001, “As praças do centro”, Expresso, supl. Revista, 23 juin, 90-92.

FERREIRA, Vítor M., 1994, "Inovação social e mudança urbana na Lisboa contemporânea: uma aproximação sucinta à inovação urbana de Lisboa, 1850-1980”, Ler História, 26: 151-170.

FRANÇA, José-Augusto, 1989, A Reconstrução de Lisboa e a Arquitectura. Lisboa, Instituto de Cultura e Língua Portuguesa.

FRANÇA, José-Augusto, 2002 [1997], Lisboa 1898: Estudo de Factos Socioculturais. Lisboa, Livros Horizonte.

GASPAR, Jorge, 1976, A Dinâmica Funcional do Centro de Lisboa. Lisboa, Livros Horizonte. GASPAR, Jorge, 1987, "Do pelourinho ao centro comercial", Povos e Culturas, 2: 243-260.

GHORRA-GOBIN, Cynthia, 2001, "Réinvestir la dimension symbolique des espaces publics", dans Cynthia Ghorra-Gobin (dir.), Réinventer le sens de la ville: Les espaces publics à l'heure globale. Paris, L'Harmattan, 5-15.

HANNERZ, Ulf, 1983 [1980], Explorer la ville. Paris, Les Editions de Minuit. 
JANEIRO, Maria J., 2006, Lisboa: Histórias e Memórias. Lisboa, Livros Horizonte.

JOSEPH, Isaac, 1995, “Reprendre la rue”, dans Isaac Joseph (dir.), Prendre place: Espace public et culture dramatique. Paris, Editions Recherches - Plan Urbain, 11-35.

LOUSADA, Maria A., 2010, "Praça”, dans Christian Topalov, Laurent Coudroy de Lille, Jean-Charle Depaule et Brigitte Marin (dirs.), L'Aventure des mots de la ville: à travers le temps, les langues, les sociétés. Paris, Robert Laffont, 979-984.

MACHADO, Fernando L., 1994, "Luso-africanos em Portugal: nas margens da etnicidade", Sociologia: Problemas e Práticas, 16: 11 1-134.

MALET, Daniel, 2011 , "A Ver Quem Passa”: O Rossio, Proceso Social y Dinámicas Interactivas en Una Plaza del Centro de Lisboa. Barcelona, Universitat de Barcelona, thèse de doctorat en Anthropologie Sociale et Culturelle.

PIRES, Rui, 2000, "O regresso das colónias", dans História da Expansão Portuguesa, vol. 5. Lisboa, Temas e Debates, 182-196.

PIRES, Rui, 2003, Migrações e Integração: Teoria e Aplicações à Sociedade Portuguesa. Oeiras, Celta Editora.

SAlGADO, Manuel, et Nuno LOURENÇO (dirs.), 2006, Atlas Urbanístico de Lisboa. Lisboa, Argumentum.

SALGUEIRO, Teresa Barata, 1997, "Lisboa: metrópole policêntrica e framentada”, Finisterra, 63: 179-190.

SAlgueiro, Teresa Barata, 2001, Lisboa: Periferia e Centralidades. Oeiras, Celta Editora.

SANTANA, Francisco, et Eduardo SUCENA, 1994, Dicionário da História de Lisboa. Lisboa, Carlos Quinta e Associados.

SANTOS, Maria do R. (dir.), 1990, Rocio-Rossio: Terreiro da Cidade. Porto, Edições Asa, CML - Arquivo Fotográfico.

SEIXAS, João, 2005, "A Baixa pombalina: análise sócio-económica de um centro mercantil europeu no início do século XXI", dans João Mateus (dir.), Reabilitação Urbana, Baixa Pombalina: Bases para Uma Intervenção de Salvaguarda. Lisboa, CML - Licenciamento Urbanístico e Reabilitação Urbana, 69-87.

SILVA, Carlos, 1994, Política Urbana em Lisboa. Lisboa, Livros Horizonte.

SIMMEL, Georg, 1981 [1970], Sociologie et Épistémologie. Paris, Presses Universitaires de France.

VALE, Teresa L. (dir.), 2008, A Cidade Pombalina: História, Urbanismo e Arquitectura, os 250 Anos do Plano da Baixa - Actas das Jornadas. Lisboa, Câmara Municipal de Lisboa.

VIEGAS, Inês, et Miguel MARTINS (dirs.), 1998, Do Passeio à Avenida: Os Originais do Arquivo Municipal de Lisboa. Lisboa, CML - Departamento do Património Cultural.

VIERA, Maria del Carmen, 1998, La Cultura de lo Cotidiano: Estudio Sociocultural de la Ciudad de Lugo. Madrid, Akal. 